\title{
Superconducting wire subject to synchronous oscillating excitations: power dissipation, magnetic response, and low-pass filtering
}

\author{
H. S. Ruiz, ${ }^{1}$ a) A. Badía-Majós, ${ }^{1}$ Y. A. Genenko, ${ }^{2}$ H. Rauh, ${ }^{2}$ and S. V. Yampolskiii ${ }^{2}$ \\ 1) Departamento de Física de la Materia Condensada and \\ Instituto de Ciencia de Materiales de Aragón (ICMA), \\ Universidad de Zaragoza-CSIC, María de Luna 3, E-50018 Zaragoza, \\ Spain \\ 2) Institut für Materialwissenschaft, Technische Universität Darmstadt, \\ Petersenstrasse 32, D-64287 Darmstadt, Germany
}

(Dated: 22 February 2012)

Numerical simulations of a type II superconducting wire subject to an ac transport current and oscillating transverse magnetic field are performed using the critical state theory. Time-dependent distributions of the current and the density of magnetic flux, the local power dissipation, and cycles of the magnetic moment are displayed. Noticeable non-homogeneous dissipation and field distortions are revealed. Results for hysteretic ac losses are reported too, and significant differences to predictions of available approximate formulae identified. Finally, a distinct low-pass filtering effect intrinsic to the wire's magnetic response is revealed.

PACS numbers: $74.25 . \mathrm{Sv}, 74.25 . \mathrm{Ha}, 41.20 . \mathrm{Gz}, 02.30 . \mathrm{Xx}$

a)Electronic address: hsruizr@unizar.es 
FIG. 1. (Color online): Sketch of the electromagnetic observables derived from our simulations. Units are $\left(\mu_{0} / 4 \pi\right) R J_{c}$ for $B_{y}, I_{c} \equiv \pi R^{2} J_{c}$ for $I_{t r},\left(\mu_{0} / 4 \pi\right) R^{2} J_{c}^{2} / \delta t$ for $\mathbf{E} \cdot \mathbf{J}$, and $R^{3} J_{c}$ for $M_{y}$. Here, the displayed frame corresponds to the external flux density $B_{y}=-0.64$, and transport current $I_{t r}=-0.24$, for the synchronous triangular excitations centrally displayed, with amplitudes $B_{y}^{\max }=2$, and $I_{t r}^{\max }=0.75$. See Ref.2 for a detailed explanation of the different panes (enhanced online).

Practical configurations of type-II superconducting wires exhibit complicated nonlinear and hysteretic behavior in oscillating electromagnetic fields. Thus, with a view to future power applications, considerable effort has been made to grasp the factors that determine the ac losses of such wires under typical operating terms. Major features of their macroscopic electromagnetic behavior have been captured in Bean's model of the critical state. ${ }^{1}$ Magnetization currents induced within the superconductor during variations of the magnetic field redistribute themselves across the sample, so as to screen the penetrating flux with their density adopting the critical value $J_{c}$ at a given temperature and specified field. Although simple for idealized configurations, the electrodynamics underlying Bean's model becomes cumbersome when realistic configurations are addressed.

In this letter, we consider the synchronous action of an impressed ac transport current $I_{t r}(t)$ directed along the main axis, $z$-axis, of an infinite type-II superconducting cylinder of radius $R$ and cross section $\Omega$, and a simultaneous transverse magnetic field of flux density $\mathbf{B}_{0}(t)=\left(0, B_{y}, 0\right)$ as sketched in Fig. 1 . Such a configuration may serve as a prototype element of large magnetic coils in which each turn is affected by its neighboring constituents. This translational invariant, two-dimensional system implies the following complexity. Due to the change with time of the excitations, a sort of free-boundary problem is conventionally solved: variations of the magnetic flux penetrate from the wire's surface, marking off the evolutionary profile of the flux front. Determining such a front, or the core within, is a tough mathematical challenge, which has been met by several approaches before. ${ }^{3-13}$ In particular, front-tracking methods are hard to use owing to the intricate dynamics of the flux front, which may even split into multiply connected domains. Here, we follow the most popular trend in the analysis of electromagnetic applications, viz. numerical simulations implementing finite-element techniques. The latter method works without explicit inclusion 
FIG. 2. (Color online) Hysteretic ac losses per cycle for synchronous ac magnetic flux density and oscillating transport current of amplitudes $\left(B_{y}^{\max }, I_{\mathrm{tr}}^{\max }\right)$. Results of this work are shown as color solid lines with markers. Comparisons with results from conventional approaches are shown for, (i) Left pane: separate excitations $L\left(B_{y}^{\max }\right)$ (black solid line) and $L\left(I_{\mathrm{tr}}^{\max }\right)$ (straight color lines), as well as their linear superposition $L\left(B_{y}^{\max }\right)+L\left(I_{\mathrm{tr}}^{\max }\right)$ (color dashed lines); (ii) Central pane: an ac magnetic field together with a dc transport current of intensity $I_{\mathrm{tr}}^{\mathrm{dc}}=I_{\mathrm{tr}}^{\max }, L\left(B_{y}^{\max }, I_{\mathrm{tr}}^{d c}\right) ;(i i)$ Right pane: the whole set of results is also plotted in linear scale. Units for losses are $\left(\mu_{0} / 4 \pi\right) \omega R^{2} J_{c}^{2}$.

of the (as yet unknown) boundary: the computations involve the entire superconducting domain, furnishing the boundary as part of the solution itself. Then, taking advantage of this methodology, we report on systematic investigations of the electromagnetic behavior of the superconducting wire. In particular, we establish (i) the geometry of local power dissipation, (ii) the need for thoughtful application of some standard approximations relating to hysteretic ac losses understood as an averaged physical quantity, and (iii) a distinct lowpass filtering effect revealed in the wire's magnetic response.

Finite-element simulations proceed from a discretization of the wire's cross section $\Omega$ by a collection of parallel cylindrical filaments $i$, each of them contributing the current density $J_{i}$ in their respective cross section $\Omega_{i}$ and carrying the partial current $I_{i}$ to make up the total transport current $\sum_{i} I_{i}=I_{t r}$, which is bound by the upper limit $I_{c}=\pi R^{2} J_{c}$. We solve Faraday's law $\delta \mathbf{B}=-\nabla \times \mathbf{E}(\mathbf{J}) \delta t$ iteratively, relating the change of the magnetic flux density $\delta \mathbf{B}$ to the electric field $\mathbf{E}$ after an increment of time $\delta t .{ }^{14}$ Here, we recall that $\mathbf{B}$ includes the external source as well as the response of the superconductor. Following Bean, the restriction imposed on the (only non-zero) current density component longitudinal to the wire thereby reads $\left|J_{z}\right| \leq J_{c}$. An equivalent would be the use of the current-field relation $E_{z}\left(J_{z}\right)=\rho J_{z}$, with the electrical resistivity $\rho\left(J_{z}\right)=0$ for $\left|J_{z}\right| \leq J_{c}$ and $\rho\left(J_{z}\right) \rightarrow \infty$ if $\left|J_{z}\right|>J_{c}$. In the quasistatic regime, the discrete form of Faraday's law obtains by a purely spatial variational principle. ${ }^{15}$ Thus, upon averaging the electromagnetic field Lagrangian within each interval of time, one ends up minimizing the volume integral of the quantity $(1 / 2)[\mathbf{B}(t+\delta t)-\mathbf{B}(t)]^{2}$, coupling successive time layers. ${ }^{14,15}$ Constraints originate from the excitations and from the chosen material law. Eventually, on introducing the vector potential A to represent the density of the magnetic flux, the quantity to be minimized at 
time $t+\delta t$ transforms into

$$
\frac{1}{2} \sum_{i, j} I_{i} \mathcal{M}_{i j} I_{j}-\sum_{i, j} \tilde{I}_{i} \mathcal{M}_{i j} I_{j}+\sum_{i} I_{i}\left(A_{z}^{e}-\tilde{A}_{z}^{e}\right),
$$

where $A_{z}^{e}$ means the (only non-zero) vector potential component related to the applied magnetic field, and $\mathcal{M}_{i j}$ denotes the inductance between (unknown) partial currents $I_{i}$, $I_{j}$ flowing through the filaments $i, j$. The tilded quantities concerning previous time $t$. Computations, with $I_{t r}$ supplied, have been performed resorting to specialized large-scale constrained minimization algorithms described elsewhere. ${ }^{14}$

In the numerical analysis, a regular grid of identical filaments, of radius $a \ll R$, located at positions $\mathbf{r}_{i}$ has been assumed. For a sufficiently refined mesh, the current density within each of the filaments will be almost uniform, which allows the magnetic vector potential contribution $A_{i, z}$ due to current flow to be readily assessed. ${ }^{15}$ We note that an arbitrary constant may be added to this quantity, leaving the magnetic field created by the filaments unchanged. Its zero choice - safe in the absence of charge transport - entails the selfinductance $\mathcal{M}_{i i}=\mu_{0} / 8 \pi$ and mutual inductance $\mathcal{M}_{i j}=-\left(\mu_{0} / 2 \pi\right) \ln \left(d_{i j} / a\right)$, with $\mu_{0}$ the permeability of vacuum and the inter-filamentary distance $d_{i j}=\left|\mathbf{r}_{i}-\mathbf{r}_{j}\right|$.

Evaluations of the electric field in the presence of current flow may, however, be tampered if the ambiguity of the magnetic vector potential is ignored. Thus, the electric field must generally be expressed as $\mathbf{E}=-\partial_{t} \mathbf{A}-\nabla \varphi$, including an electrostatic-like term derived from a scalar potential $\varphi$. For the wire at hand, $\nabla \varphi$ should be uniform in space, permitting the representation $E_{z}=-\partial_{t} A_{z}+C(t) \equiv \partial_{t} A_{z}^{\prime}$, with the calibrated vector potential component $\partial_{t} A_{z}^{\prime} \cdot{ }^{16}$ Since $A_{z}$ is only determined up to an additive constant for each increment of time, the situation may be tackled by calling upon the displayed expression and progressively resolving $C(t)$ in accordance with the physical condition $E_{z}=0$ at those points where magnetic flux does not change.

Simulations have been performed for the triangular oscillating process displayed in Fig. 1. The following quantities have been focused on: (i) magnetic field lines derived from $\mathbf{B}=$ $\nabla \times \mathbf{A}$, (ii) the apparent sample's magnetic moment (per unit length of wire and normal to its axis) $\mathbf{M}=\int_{\Omega} \mathbf{r} \times \mathbf{J}$, (iii) the local density of power dissipation $(\mathbf{E} \cdot \mathbf{J})$, and the hysteretic ac losses per unit time and volume for cyclic excitations of frequency $\omega$ ensuing the spaceand time-averages $L=\left(\omega / 2 \pi^{2} R^{2}\right) \oint_{f . c .} d t \int_{\Omega} \mathbf{E} \cdot \mathbf{J}$. Here, f.c. denotes a full cycle of the time-varying electromagnetic sources. Our results and conclusions are developed along the 
FIG. 3. (Color online) Magnetic moment component $M_{y}$ relative to $\left|M_{y}\left(B_{p}, 0\right)\right|=(2 / 3)$ in the case of the synchronous ac transport current and oscillating magnetic field of amplitude $\left(B_{y}^{\max }, I_{t r}^{\max }\right)$, represented by its dependence on the sources (left) $B_{y}$ and $I_{t r}$ [scaling the x-axis according to $\left.\left.I_{t r}^{\max }\left(B_{y} / B_{y}^{\max }\right)\right)\right]$, and also by its temporal evolution (right). Plots are done for $B_{y}^{\max }=1,2,4,6$, and 8 (colored lines), as well as for the values $I_{\mathrm{tr}}^{\max }=0.25,0.5,0.75$, and 1.0 (by rows).

following paragraphs.

Fig. 1 presents a a snapshot for the main electromagnetic quantities at the time frame spotted in the central plot, for synchronous ac sources $B_{y}$ and $I_{t r}$. The detailed evolution with time of the local distribution of current density $J_{z}$, the lines of magnetic flux density $\mathbf{B}$, and the local density of power dissipation $\mathbf{E} \cdot \mathbf{J}$, as well as the dynamics of the magnetic moment component $M_{y}$ for the wire in a cyclic process can be followed in the video attached. Interestingly, the inner region of the wire (green), which is devoid of electric current and magnetic flux, and the adjacent 'active' regions (red, blue), where $J_{z}$ alternates between $J_{c}$ and $-J_{c}$, strongly depart from axial symmetry due to the consumption of the magnetization currents by effect of transport. Concomitantly, substantial distortions of the magnetic flux density outside the wire appear. These are particularly marked when $B_{y}$ and $I_{t r}$ tend to zero during excitation. The density of power dissipation, which rises from low-value parts (blue) to high-value parts (red), is highly localized too. Its pronounced bias unfolding across the wire could increase the probability of quench. From the cycles of $M_{y}$, it furthermore appears that a proper determination of the 'active' regions depends on the history of the virgin branch, thus bearing witness to the system's memory. For example, a positive slope in both $B_{y}$ and $I_{t r}$ constitutes increasing power dissipation in the positive $x$-direction perpendicular to the wire.

Fig. 2 shows the variation of the hysteretic ac losses in terms of the amplitude of the synchronous oscillating sources, $L\left(B_{y}^{\max }, I_{\mathrm{tr}}^{\max }\right)$. Our results are compared to those obtained from several analytical approximations ${ }^{6}$ customarily applied for simplified configurations. Thus, we recall the equation 
$L\left(B_{\mathrm{y}}^{\max }, I_{t r}^{d c}\right) \equiv \frac{8 B_{p}^{2}}{3 \mu_{0}} \begin{cases}\left(\frac{B_{\mathrm{y}}^{\max }}{B_{p}}\right)^{3}\left(1-\frac{B_{\mathrm{y}}^{\max }}{2 B_{p}}\right) & , \quad B_{\mathrm{y}}^{\max }<B_{p}(i) \\ {\left[\frac{B_{p}(i)}{B_{p}}\right]^{3}\left[1-\frac{B_{p}(i)}{B_{p}}\right]+\left(1+i^{2}\right) \frac{B_{\mathrm{y}}^{\max }-B_{p}(i)}{B_{p}}, \quad B_{y}^{\max }>B_{p}(i),}\end{cases}$

where

$$
B_{p} \equiv(2 / \pi) \mu_{0} J_{c} R \quad ; \quad i \equiv I_{t r}^{d c} / I_{c} \quad ; \quad B_{p}(i) \equiv\left(1-i^{2 / 3}\right) B_{p}
$$

Eq.(2) is valid for an ac magnetic flux of amplitude $B_{\mathrm{y}}^{\max }$ and an imposed de transport current $I_{t r}^{d c}$. It can be used either for evaluating the non-coupled dependence $L\left(B_{\mathrm{y}}^{\max }\right)$ or the ac/dc coupling $L\left(B_{\mathrm{y}}^{\max }, I_{t r}^{d c}\right)$. Comparisons reveal the important fact that a naive linear superposition of contributions due to either type of excitation is only appropriate for high strengths of the magnetic field and moderate, or low, currents; a finding which adds to previous work dealing with the infinite slab, ${ }^{17}$ or rectangular strip geometry ${ }^{18}$ and sheds new light on the validity of approximate formulae at the same time. Consequently, approximations such as $L\left(B_{y}^{\max }\right)+L\left(I_{t r}^{\max }\right)$ and $L\left(B_{y}^{\max }, I_{t r}^{d c}\right)$ can drastically under- or overestimate the true losses. Furthermore, additional refinements, such as that suggested in Ref.19, that proposes a time-averaging over the cycle of the expressions with $I_{t r}^{d c}$ are clearly insufficient. Notice that this would just produce a shift in the dependence predicted by the above equation that only serves for improving the estimation at high fields.

We note that for small values of $B_{y}^{\max }\left(\right.$ e.g. $\left.B_{y}^{\max }=1\right)$, the differences between the losses predicted by our numerical simulations and those obtained by the plain superposition can exceed $100 \%$ when $I_{t r}^{\max }=0.25$, but decrease to $\approx 15 \%$ as $I_{t r}^{\max }$ rises to $\approx 1$. On the other hand, when $B_{y}^{\max } \gtrsim B_{p}$, bringing in the full penetration field for zero transport current, $B_{p}$, the difference can vary between $\approx 20 \%$ for $I_{t r}^{\max } \cong 0.25$ and $\approx 55 \%$ for $I_{t r}^{\max } \cong 1$, or even more. In general, for the extensive set of simulations performed, deviations up to $\approx 100 \%$ can be observed around the choice $\left(B_{y}^{\max }=B_{p} / 2, I_{t r}^{\max }=I_{c} / 2\right)$. This demonstrates that a proper evaluation of hysteretic ac losses in the presence of synchronous electromagnetic excitations requires sophisticated analysis resources, even for a relatively simple configuration like the one studied here.

Fig. 3 shows the dynamics of the magnetic moment component $M_{y}$ for a range of values of the amplitudes of the electromagnetic excitations, $\left(B_{y}^{\max }, I_{t r}^{\max }\right)$. We realize that only 
for small values of the amplitude of the ac transport current, almost Bean-like loops of $M_{y}$ obtain. In particular, we recall a progressive disappearance of the flat saturation behavior for high values of the amplitude of the oscillating magnetic field, as $I_{t r}^{\max }$ augments. Remarkably, this phenomenon ends up with a symmetrization of the loops, both as functions of $B_{y}$ and $I_{t r}$, into characteristic lenticular shapes. As a consequence of this process, a distinct lowpass filtering effect comes to the fore which, in the case of the triangular input excitations considered here, yields a nearly perfect sinusoidal (first-harmonic) output signal $M_{y}(t)$.

In conclusion, we have examined the electromagnetic response of a straight, infinite, cylindrical type-II superconducting wire subject to synchronous oscillating excitations by means of a numerical implementation of Bean's model of the critical state. The cardinal assumption underlying our simulations thus is a current-field relation with an infinite discontinuity at the critical current density. For basic configurations, this is known to lead to (rate-independent) hysteretic ac losses which are describable by elementary relations between monotonic and cyclic quantities. We have shown that even for the plain configuration studied here, intriguing phenomena may emerge, unexpected in simplified models based on linear superposition. Thus, we have found strong distortions of the magnetic flux density outside the wire, pronounced localization effects in the density of power dissipation, and have identified important failures of approximate formulae for hysteretic ac losses; finally, we have predicted a distinct low-pass filtering effect in the wire's magnetic response, which may lead to envisage new applications for superconducting systems of the above sort.

Funding of this research by Spanish CICyT and FEDER programs (MAT2008-05983C03-01 project), DGA grant T12/2011, and Spanish CSIC JAE program is gratefully acknowledged.

\section{REFERENCES}

${ }^{1}$ C. P. Bean, Phys. Rev. Lett. 8, 250 (1962); Rev. Mod. Phys. 36, 31 (1964).

${ }^{2}$ Top-Left. The magnetic field lines (isolevels of the vector potential over the wire's crosssection) and their corresponding profiles of current density are shown. The consumption of local magnetization currents and the generated field distortions around the superconducting wire are visualized. Top-Right: Dynamics of the density of power dissipation across the section of the superconducting wire. A clear non symmetric distribution of the heat 
production in a cyclic process is always observed. Bottom-Left: The magnetization loop for the superconducting wire in terms of the applied flux density (in normalized units $\left.\left(B_{y} / B_{y}^{\max } \cdot I_{t r}^{\max }, I_{t r}^{\max }\right)\right)$ is shown. Bottom-Right: The magnetic moment is now displayed as a function of time, showing a distinct low-pass filtering effect that extracts the first harmonic of the triangular input.

${ }^{3}$ M. Ashkin, J. Appl. Phys. 50, 7060 (1979).

${ }^{4}$ M. Ashkin and G. R. Wagner, J. Appl. Phys. 60, 2477 (1986).

${ }^{5}$ W. J. Carr, Jr., AC Loss and Macroscopic Theory of Superconductors (Gordon \& Breach, New York, 1983).

${ }^{6}$ A. V. Gurevich, R. G. Mints, and A. L. Rakhmanov, Physics of Composite Superconductors (Begell House, New York, 1997).

${ }^{7}$ C. Y. Pang, A. M. Campbell, and P. G. McLaren, IEEE Trans. Mag. 17, 134 (1981).

${ }^{8}$ K. L. Telschow and L. S. Koo, Phys. Rev. B 50, 6923 (1994).

${ }^{9}$ Y. E. Kuzovlev, JETP Lett. 61, 1000 (1995).

${ }^{10}$ F. Gömöry, R. Tebano, A. Sánchez, E. Pardo, C. Navau, I. Husek, F. Strycek, and P. Kovac, Supercond. Sci. Technol. 15, 1311 (2002).

${ }^{11}$ B. ten Haken, J. J. Rabbers, and H. H. J. ten Kate, Physica C 377, 156 (2002).

${ }^{12}$ D. Karmakar and K. V. Bhagwat, Physica C 398, 20 (2003).

${ }^{13}$ L. Rostilla, S. Brisigotti, and G. Grasso, J. Supercond. Nov. Magn. 24, 313 (2011).

${ }^{14}$ A. Badía-Majós, C. López, and H. S. Ruiz, Phys. Rev. B 80, 144509 (2009); H. S. Ruiz and A. Badía-Majós, Supercond. Sci. Technol. 23, 105007 (2010); H. S. Ruiz, C. López, and A. Badía-Majós, Phys. Rev. B 83, 014506 (2011).

${ }^{15}$ A. Badía-Majós, Am. J. Phys. 74, 1136 (2006).

${ }^{16}$ F. Gömöry, M. Vojenčiak, E. Pardo, and J. Šouc, Supercond. Sci. Technol. 22, 034017 (2009).

${ }^{17}$ R. Hancox, Proc. IEE, 113, 1221 (1966).

${ }^{18}$ E. Pardo, F. Gömöry, J. Šouc, and J. M. Ceballos, Supercond. Sci. Technol. 20, 351 (2007).

${ }^{19}$ A. M. Campbell, Cryogenics 22, 3 (1982). 